\title{
GAIA Level 1 Neonatal Death in a Non- viable Live Birth
}

National Cancer Institute

\section{Source}

National Cancer Institute. GAIA Level 1 Neonatal Death in a Non-viable Live Birth. NCI

Thesaurus. Code C127969.

GAIA Level 1 Neonatal Death in a Non-viable Live Birth is defined by three criteria: first, the infant must be live born; second, at least one of the following requirements must be met: a) Gestational age less than 22 weeks (a GA level of certainty of one); OR b) Birth weight less than 500g; third, the death of the infant within the first 28 days of life must be documented. 